receive rifaximin $400 \mathrm{mg}$ every $8 \mathrm{~h}$ for 10 days $(n=32)$ or placebo $(n=20)$, and undertook a second breath test 1 month later.

The prevalence of SIBO was significantly higher in patients with rosacea than in controls $(P<0.001)$. Among the $28(87.5 \%)$ SIBO-positive patients with rosacea in whom bacterial overgrowth was eradicated by rifaximin treatment, cutaneous lesions cleared completely in 20 (71.4\%) patients and $6(21.4 \%)$ patients showed an improvement in the number of papules and papulopustules. The eradication of SIBO was also associated with a significant improvement in gastrointestinal symptoms in these individuals. Cutaneous lesions were either unchanged or worse in SIBO-positive patients with rosacea who received placebo. At 9-month follow-up cutaneous signs of rosacea remained in remission in $96 \%$ of SIBO-eradicated patients.

The authors conclude that these results strongly support a pathogenic role of intestinal bacteria and their products in the development of cutaneous lesions in rosacea.

Original article Parodi A et al. (2008) Small intestinal bacterial overgrowth in rosacea: clinical effectiveness of its eradication. Clin Gastroenterol Hepatol 6: 759-764

\section{GERD: PPIs decrease immune response, but not DNA damage}

Whether PPI therapy can prevent Barrett's esophagus and esophageal adenocarcinoma is controversial: studies have produced contradictory results. Researchers in the Netherlands determined the esophageal immune response and prevalence of DNA adducts in patients with GERD, and investigated the effect of PPI therapy on these parameters. They found that, although PPI therapy reduces the cellular immune response, it does not affect the number of DNA adducts in the esophageal epithelium of patients with erosive esophagitis (EE).

The study by De Jonge et al. included 40 patients with GERD: 25 had EE and 10 had nonerosive reflux disease (NERD). The inflammatory infiltrate in esophageal biopsy samples consisted mainly of T cells: both the number of $\mathrm{T}$ cells and the proportion of $\mathrm{CD} 8^{+} \mathrm{T}$ cells were significantly higher in patients with EE than in patients with NERD. T-cell numbers correlated significantly with endoscopic esophagitis grade. Twenty patients with EE were randomly allocated to 4 weeks or 8 weeks of rabeprazole therapy; in both groups, the numbers of $\mathrm{T}$ cells, $\mathrm{CD} 8^{+}$ $\mathrm{T}$ cells, mast cells and macrophages decreased significantly after treatment.

The prevalence of DNA adducts was measured in a subset of patients ( 11 with NERD and 17 with $\mathrm{EE})$. The level of inflammation did not correlate with the number of adducts, which was similar in NERD and EE patients. Rabeprazole therapy for 4 or 8 weeks in EE patients did not reduce the prevalence of DNA adducts in esophageal biopsy samples.

The findings suggest that short-term PPI therapy reduces the immune response in patients with GERD, but might not protect against esophageal cancer.

Original article de Jonge PJF et al. (2008) Proton pump inhibitor therapy in gastro-oesophageal reflux disease decreases the oesophageal immune response but does not reduce the formation of DNA adducts. Aliment Pharmacol Ther 28: 127-136

\section{Good outcomes for pancreatectomy with IAT in children with chronic pancreatitis}

A new study has revealed benefits for pancreatectomy with islet autotransplantation (IAT) in pediatric patients with chronic pancreatitis. Bellin and colleagues demonstrated that this combination treatment not only eliminates or reduces pain but also preserves insulin secretory capacity to prevent or minimize the otherwise inevitable post-pancreatectomy diabetes mellitus in this population.

The researchers retrospectively reviewed medical records and attempted to contact all 24 patients who had undergone pancreatectomy with IAT at the University of Minnesota Medical Center when aged $<19$ years. Preoperatively, all patients required narcotic medications, and $75 \%$ required chronic narcotic use. Of the 18 patients for whom follow-up data was available, $61 \%$ had discontinued all narcotic pain medication, while $67 \%$ of surviving patients reported no pain symptoms and $27 \%$ reported pain improvement. At 1 year or more after transplant, $56 \%$ of patients were insulin-independent, while a further $22 \%$ retained partial graft function-they required insulin only once daily.

Interestingly, pre-adolescent patients $(<13$ years old) were significantly more likely than 